\title{
A COUNTEREXAMPLE TO CONJECTURES OF PAPAKYRIAKOPOULOS AND SWARUP ${ }^{1}$
}

\author{
JAMES MCCOOL
}

\begin{abstract}
In [5] Swarup states a group theoretic conjecture P2 and shows that $\mathbf{P 1} \Rightarrow \mathbf{P} 2 \Rightarrow$ the Poincare conjecture, where $\mathbf{P 1}$ is a conjecture of Papakyriakopoulos [3]. We give a counterexample to conjecture $\mathbf{P 2}$.
\end{abstract}

In [3], Papakyriakopoulos stated a conjecture P1 and showed that P1 implies the Poincaré conjecture. In [5], Swarup showed that $\mathrm{P} 1 \Rightarrow \mathrm{P} 2 \Rightarrow$ the Poincaré conjecture, where $\mathbf{P} 2$ is the following.

ConJecture. Let $Q_{p}=\left\{a_{1}, b_{1}, \ldots, a_{p}, b_{p} ; \Pi_{i=1}^{p}\left[a_{i}, b_{i}\right],\left[a_{1}, b_{1} \tau\right]\right\}$, where $p>1$ and $\tau \in\left[\Phi_{P}, \Phi_{P}\right], \Phi_{P}$ being the free group on $a_{1}, b_{1}, \ldots, a_{p}, b_{p}$. Then $Q_{P}$ is a nontrivial free product. In this note we show, by means of a counterexample, that conjecture $\mathrm{P} 2$ is false. We take $p=2, \tau=\left[a_{1}, b_{1}\right]$ and suppose that $Q_{2}=A * B$, where $A$ and $B$ are nontrivial. Now we observe that, with a slight change of notation,

$$
\begin{aligned}
Q_{2} & =\{a, b, c, d ;[a, b][c, d],[a, b a b \bar{a} \bar{b}]\} \\
& =\{a, b, c, d, x, y ; b a \bar{b} \bar{x}, b x \bar{b} \bar{y}, a b \bar{a} \bar{b}[c, d], a b a b \bar{a} \bar{b} \bar{a} b a \bar{b} \bar{a} \bar{b}\} \\
& =\{a, b, c, d, x, y ; b a \bar{b} \bar{x}, b x \bar{b} \bar{y}, a \bar{x}[c, d], a x \bar{y} \bar{x} y \bar{x}\} \\
& =\left\{b, c, d, x, y ; b x \bar{y} x y \bar{x} \bar{b} \bar{x}, b x \bar{b} \bar{y}, x \bar{y} x y \bar{x}^{2}[c, d]\right\} \\
& =\left\{b, c, d, x, y, z ; \bar{x} x \bar{y}, b x \bar{y} x y \bar{x} \bar{b} \bar{x}, b x \bar{b} \bar{y}, x \bar{y} x y \bar{x}^{2}[c, d]\right\} \\
& =\{b, c, d, x, z ; b z x \bar{z} \bar{b} \bar{x}, b x \bar{b} \bar{x} z, z x \bar{z} \bar{x}[c, d]\} .
\end{aligned}
$$

Let $K=\{z, x, c, d ;[z, x][c, d]\}$. Then using the Freiheitssatz (see [2] or [1]), we see that the subgroup of $K$ generated by $x, z$ is free of rank two, and so therefore are the subgroups generated by $z x \bar{z}, x$ and $x, \bar{z} x$, respectively. It follows that $Q_{2}$ is an $H N N$-extension (see, e.g., [1]) of $K$ by the stable letter $b$, and hence that the subgroup of $Q_{2}$ generated by $z, x, c, d$ is naturally isomorphic to $K$. Now $K$ is not cyclic, and from the results of [4] $K$ is indecomposable with respect to free products. It then follows from the Kurosh Subgroup Theorem that $K$ is contained in a conjugate of $A$ or $B$, say $B$. By conjugating the original $A$ and $B$, we may assume that $K$ is contained in $B$.

Received by the editors October 25, 1979.

1980 Mathematics Subject Classification. Primary 20E06; Secondary 57M40.

'This work was supported by a grant from the Natural Sciences and Engineering Research Council, Canada. 
Since $b$ and $K$ generate $Q_{2}$, it follows that $b \notin B$. Now the second relator of the last presentation of $Q_{2}$ above gives $b x \bar{b}=\bar{z} x$, i.e. conjugation of the element $x$ of $B$ by $b$ gives the element $\bar{z} x$ of $B$. Consideration of the normal form of $b$ shows immediately that this implies $b \in B$, which is a contradiction.

Thus $Q_{2}$ must be indecomposable with respect to free products.

\section{REFERENCES}

1. R. C. Lyndon and P. Schupp, Combinatorial group theory, Springer-Verlag, Berlin and New York, 1977.

2. W. Magnus, A. Karrass and D. Solitar, Combinatorial group theory, Wiley, New York, 1966.

3. C. D. Papakyriakopoulos, A reduction of Poincaré conjecture to group theoretic conjectures, Ann. of Math. (2) 77 (1963), 250-305.

4. A. Shenitzer, Decomposition of a group with a single defining relation into a free product, Proc. Amer. Math. Soc. 6 (1955), 273-279.

5. G. A. Swarup, Two reductions of the Poincaré conjecture, Bull. Amer. Math. Soc. N.S. 1 (1979), 774-777.

Department of Mathematics, University of Toronto, Toronto M5S 1A1, Ontario, Canada 